\title{
O Registro Clínico Computadorizado
}

\author{
Adilton Lobo \\ Mestre em Ciência da Computação (Universidade Federal de SC) Florianópolis - SC - 2003. \\ Área de Concentração do Mestrado: Sistemas de Conhecimento. \\ Joinville,SC - maio de 1999
}

\begin{abstract}
Resumo
O artigo dispõe sobre o Registro Clínico Computadorizado e inicia com o emprego da informática na medicina, fala sobre a relação médico-computador e da origem, necessidade e importância do Registro Clínico. Mostra como o Registro Clínico Computadorizado pode integrar os diversos setores hospitalares e o seu papel de informar, integrar e agilizar o atendimento ao paciente e as vantagens de sua implantação em segmentos médicos e administrativos. Idealizou-se uma rede onde estes registros circulariam, provendo informações em todos os "nós" - terminais e ao Sistema Público de Saúde. E ainda o uso de smart-cards, exemplificando suas aplicações nos dias de hoje, em países que adotaram a tecnologia como forma de demonstrar um bom atendimento ao paciente, preocupação com a redução e controle de custos e fraudes e busca sincera da melhoria da qualidade de vida de seus povos.
\end{abstract}

Palavras-chave: Registro Clínico, registro clínico computadorizado, smart-card, sistema público de saúde

\begin{abstract}
This article presents the computorized clinical records and the beginnings of medical informatics, treats the relation physician-computer, the origins, necessity and importance of good clinical records. The integration of several sectors of the hospital can be integrated by using the computer as a tool for a health data base, showing its utility in patient care and administrative issues. It is possible to use a network and the clinical records can be available in different nodes of the network, where a terminal of the Public Health System needs. The so called smart cards, for example, that are widely applied in countries that adopted this technology has shown the good quality of patient care, reduction and control of costs and frauds, contributing to an increase of life quality.
\end{abstract}

\section{Introdução}

O emprego da informática na área médica confunde-se com o próprio início da utilização dos computadores. As 
primeiras aplicações na área hospitalar ou de registro clínico remontam-se à década de 60, quando surgiram o Technicom Medical Information System (TMS), o COSTAR e, na década seguinte, os sistemas HELP, PROMIS e Regenstrief Medical Record System.

Se, no início, eram poucas as instituições que podiam lançar mão desta tecnologia, devido ao alto custo dos computadores de grande porte, logo o avanço tecnológico e o conseqüente barateamento dos equipamentos permitiu que até mesmo os departamentos passassem a adquirir minicomputadores para informatização de suas rotinas. Esta realidade passou a se tornar uma constante a partir do final da década de 70 e início dos anos 80. Esta foi também a época da consolidação dos grandes sistemas de informação hospitalar, que tinham por objetivo a automatização de todas as rotinas administrativas de um hospital. Já no final dos anos 80, o avanço tecnológico também permitiu que até mesmo microcomputadores viessem a fazer parte do arsenal utilizado pelos hospitais para a informatização de seus serviços. Surgiram então hospitais totalmente informatizados, com terminais individuais para cada leito (bedside terminals). Os maiores beneficiados com esta nova ferramenta foram as pequenas instituições e mesmo clínicas privadas, as quais puderam assim viabilizar economicamente o emprego de computadores.

A análise inicial do quadro acima dá margem a que se imagine a existência de um grande avanço na qualidade da assistência prestada aos pacientes. No entanto, é necessário esclarecer que, principalmente em ambientes hospitalares, a informatização tomou conta basicamente das rotinas de caráter administrativo. $\mathrm{O}$ computador permaneceu distante das rotinas de caráter eminentemente clínico e, por conseguinte, da mão dos médicos, salvo raras exceções.

Obviamente, mesmo a informatização de caráter administrativo traz benefícios quanto à qualidade de atendimento prestada. No entanto, não se pode deixar de lembrar que a atividade de uma instituição de saúde gira em torno dos pacientes e que estes, quando a procuram, comunicam-se diretamente com o médico para que este formule suas hipóteses diagnosticas e estabeleça um plano terapêutico adequado. Alguns pontos fundamentais advém ou devem ser aliados a esta ponderação:

O processo hipotético-dedutivo empregado pelo médico para a solução dos problemas clínicos é uma atividade fundamentalmente baseada em informação;

- A grande maioria das informações de natureza clínica são geradas a partir do encontro do médico com seu paciente;

- As instituições, por conseguinte, dependem do médico para obter informações, principalmente de caráter clínico;

- As informações dos pacientes são (ou devem ser) registradas no prontuário clínico.
Assim, é factível supor que um avanço significativo da qualidade do atendimento prestado depende da informatização do prontuário clínico e da interação do médico com o sistema, como provedor e usuário das informações.[2]

Propor-se-á nesta monografia, que estas informações dos pacientes circulem entre hospitais, laboratórios, postos de saúde. Em uma rede de micros/terminais, os profissionais da saúde, teriam acesso, aos dados clínicos pregressos do paciente em questão, com isto, teriam mais rapidez no atendimento, redução de custos (devido a exames que não teriam a necessidade de se repetir e diminuição de acidentes ou falhas médicas.

Em 1988, como conclusão dos trabalhos do "Working Group 10" da IMIA (Hospital Information Systems), ressaltava-se que os registros clínicos estavam se tornando o foco de interesse para o desenvolvimento de sistemas de informação hospitalar. Os altos custos da medicina têm chamado a atenção de um significativo segmento de pesquisadores de informática médica, que, na busca de uma solução que, mantendo ou melhorando a qualidade de atendimento, venha a evitar desperdícios, colocam como uma de suas principais metas a informatização do registro clínico. [2]

\section{O Registro Clínico}

O registro clínico é um conjunto de campos relativos às informações sobre um determinado paciente. Internamente, em um hospital, funciona acumulando dados que ficam à disposição entre os funcionários da área médica e administrativa. Sendo que, em cada setor hospitalar onde o paciente estiver gerando novas informações, lá estará sendo atualizado, acrescido, analisado, enfim utilizado seu registro.

É evidente que todos os sistemas administrativos dependem das informações provenientes dos profissionais de saúde, pois, afinal, são eles que realizam a grande maioria dos contatos com os pacientes, razão da existência da instituição como um todo. Se a interação direta destes profissionais é benéfica para o hospital, nada mais justo do que verificar o que seria necessário dar em troca para cativá-lo a aceitar esta interação.

Que vantagem teria o médico em "perder ainda mais tempo" com um computador? Este é certamente o questionamento que a maioria dos potenciais beneficiários se fazem ao se depararem com a possibilidade de ter de interagir com estas "máquinas". A maioria provavelmente é capaz de imaginar as vantagens que tal interação traria ao hospital, tais como:

- Menor necessidade de pessoal administrativo para realizar a transcrição e o processamento das solicitações médicas,

- Inexistência de erros de transcrição, 
- Provável diminuição do tempo de internação pela agilização do serviço.

Por outro lado, poucos se dão conta das vantagens pessoais que tal interação traria. Este desconhecimento não é "privilégio" ou responsabilidade dos médicos, pois os próprios administradores ou "informatas" responsáveis pela confecção e implantação de sistemas hospitalares não são capazes de avaliar as necessidades ou benefícios do ponto de vista médico e incluí-los nos seus sistemas computadorizados.

A comunicação do médico para com o hospital se faz (ou deveria ser feita) através do registro clínico do paciente que é, ou, da mesma maneira, deveria ser, a fonte central de informação a partir da qual seriam derivados todos os demais procedimentos do hospital. Por conseguinte, a interação do médico com esta "ferramenta" seria suficiente para prover o hospital das informações necessárias. As vantagens de interação podem se originar da realização de tarefas tão simples, do ponto de vista computacional, como preenchimento automático de prescrições (renovações), até tarefas mais complexas como auxílio à decisão.

Sem dúvida, qualquer destes benefícios se tornariam inúteis caso o registro clínico computadorizado não apresentasse uma interface adequada, pois a dificuldade de interação é o segundo fator limitante no envolvimento do médico. [4]

O melhor enfoque visando "proporcionar algo" para o médico é confecção do registro clínico computadorizado, já que é este o principal instrumento de registro e comunicação utilizado pelo médico como meio de interação para com o hospital .

É importante notar que os módulos dos sistemas hospitalares têm, além do valor intrínseco relativo à função que executam, o grande valor de servirem como meio de intercomunicação entre os diversos setores. Alguns autores até consideram que este é o principal papel de um sistema de informação hospitalar. [2]

Bleich, considera que a grande maioria dos sistemas até então desenvolvidos são do tipo "interfaceado" e não do tipo "integrado". As vantagens do primeiro estariam na não dependência, por parte do hospital, de um único fabricante (caso houvessem padrões de intercomunicação). Os sistemas integrados, por sua vez, baseiam-se em uma nova concepção na qual a organização é vista como um todo, cabendo aos departamentos o detalhamento dos dados exclusivos da especialidade. [1]

Além disto, sendo de concepção única, ter-se-ia como vantagem adicional a existência de um único padrão de interação para com os profissionais de saúde em todo o sistema, evitando-se assim problemas adicionais com treinamento. Esta concepção vem de encontro ao conceito de banco de dados distribuído, também mencionado por outros autores. [6]

\section{Funções e Objetivos do Registro Clínico}

Primeiramente, é necessário avaliarmos quais são as funções de um registro clínico a fim de que verifiquemos onde a computação poderia atuar de maneira decisiva para a melhoria de qualidade deste e promoção da interação dos médicos. O registro clínico tem como principais objetivos:

1. Auxiliar o tratamento dos pacientes - como instrumento onde todas as informações coletadas são depositadas, o registro clínico serve sobretudo como base para a formação de opinião sobre o(s) problema(s) de saúde do paciente, bem como para o registro do planejamento clínico a ser implementado e o seguimento do resultado destas ações. Ele pode, ainda, ser considerado como o instrumento de comunicação entre os diferentes membros da equipe médica que tratam do paciente, bem como mecanismo de memória, visando garantir a continuidade de tratamento do paciente durante a hospitalização e entre diferentes hospitalizações ou consultas ambulatoriais, bem como possibilitando avaliar o curso das doenças ao longo da vida do paciente;

2. Servir como base para as ações administrativas e avaliação de desempenho - o registro clínico é a fonte para realização de todos os procedimentos administrativos e financeiros, tais como cobrança das contas dos pacientes ou das instituições de saúde, avaliação do desempenho da equipe, verificação do grau de qualidade do atendimento prestado (média de dias de internação, incidência de infecção hospitalar) ;

3. Servir como fonte de informações para a pesquisa médica - os estudos retrospectivos para detecção de efeitos colaterais de medicamentos, avaliação do sucesso de novas terapias, etc... só podem ser feitos com base nas informações colhidas dos pacientes ;

4. Servir como documento legal de registro da atuação médica - Embora não seja uma utilização tão enfatizada a nível nacional, sempre houve preocupação com este aspecto que não é só garantia do paciente como do próprio médico ou dos demais profissionais de saúde envolvidos. [4]

\section{Automatizando o Registro Clínico}

Se até certo ponto o registro manual é capaz de desempenhar de maneira razoável as funções relativas ao atendimento do paciente em si, muito difícil tem sido sua utilização com o objetivo de dar suporte à avaliação do desempenho das instituições ou mesmo aquisição de novos conhecimentos médicos, pois estas tarefas envolvem a manipulação de todo o conjunto de registros existentes, o que só é factível de maneira automatizada.

Avaliando a nível individual, é também possível identificar algumas fraquezas, pois "as informações do 
paciente precisam estar disponíveis quando e onde necessárias", o que muitas vezes não é possível com o registro manual. Duas pessoas só poderão acessar as mesmas informações ao mesmo tempo e em lugares diferentes caso estejam disponíveis através de meio eletrônico. Da mesma maneira, resultados de exames complementares só estariam prontamente disponíveis para a utilização pelos médicos nas enfermarias caso se utilizasse um sistema computadorizado.

$\mathrm{Na}$ literatura podem ser encontradas referências sobre quais são os tipos de dados que os médicos consideram primordiais para a prática médica do dia-adia. Entre eles estão a Lista de Problemas, quer sejam sintomas, sinais, dados laboratoriais anormais, estados fisiopatológicos, diagnósticos ou problemas psicossociais; resultado de testes e exames complementares, terapia em curso e parâmetros da atividade do paciente (sinais vitais, atividade física, ingesta, perdas), bem como o resumo da última hospitalização ocorrida. O computador certamente é útil como meio de comunicação entre os diferentes setores que produzem estes dados e como ferramenta para a organização destas informações. Todavia, isto não é suficiente para que o médico encare o computador como uma ferramenta de aumento de produtividade, passível de motivá-lo a interagir no dia-a-dia. É necessário que o computador seja "suficientemente inteligente para executar automaticamente as tarefas de natureza mecânica, comportando-se como auxiliar direto do médico no suporte a suas atividades clínicas, sem com isto impor uma demanda especial para seu aprendizado ou interação". Em última análise o computador passaria a ser olhado com vantagem caso assumisse o papel do "braço-direito" do médico. [4]

\section{Vantagens do Registro Clínico Computadorizado}

É, de certa maneira, até fácil enumerar exemplos que demonstrem a clara vantagem do computador em relação a utilização das informações constantes no registro clínico por parte do médico. No entanto, não devemos esquecer o duplo papel do médico como provedor e usuário das informações. Garantir a sua interação direta para com o computador está, mais, na dependência de o auxiliarmos na primeira parte (aquisição de dados). Obviamente a segunda também desperta grande interesse, já que está intimamente ligada ao processo hipotéticodedutivo empregado no raciocínio clínico e planejamento terapêutico, a função nobre da atividade médica. A melhoria da qualidade de atendimento depende do auxílio do computador neste sentido. [4]

A primeira regra é simples: automatize a aquisição de dados do maior número de informações possíveis, evitando que o médico tenha de perder tempo com esta tarefa. Entre as informações que se enquadram nesta categoria estão o resultado de exames complementares e monitoração dos sinais, pois a maior parte dos equipamentos atualmente utilizados dispõem de saída digital permitindo sua integração com os sistemas computadorizados do hospital.

Um segundo exemplo em que o computador pode auxiliar é na aquisição de dados que são, na realidade, derivados de outros dados existentes no registro clínico. Todos os cálculos com a finalidade de produzir novas informações devem ser realizados pelo computador.

No que tange à padronização da coleta de informações, o computador é um ótimo mecanismo para forçar o cumprimento de padrões de coleta de dados, lembrando ao médico que informações são consideradas relevantes para o hospital. Assim cada especialidade poderia definir quais os seus dados mínimos, inclusive com base em diferentes quadros clínicos, permitindo que o computador alertasse o médico sobre a falta da coleta de uma informação essencial. Isto pode se desdobrar até no preenchimento automático do pedido de um exame complementar, faltando apenas a aquiescência do médico para que a ordem seja encaminhada. [4]

A informatização do registro clínico traz como primeira vantagem a apresentação limpa (datilografada) e organizada da informação. Este benefício é decorrente do próprio processo de análise da instituição, como passo anterior à informatização. Todavia, estes ganhos, em termos de apresentação da informação, são importantes e não deixam de ser lembrados pelos médicos como condição ou benefício básico para a utilização de registros clínicos computadorizados.

No entanto, ao considerarmos que é fundamental a interação direta do médico com o registro clínico computadorizado, visando um aumento da qualidade de atendimento, vários outros detalhes são necessários para tornar o computador suficientemente inteligente e, por conseguinte, desejado pelos médicos como ferramenta de trabalho no dia-a-dia.

Como "braço-direito", o computador tem de se tornar um parceiro ativo no processo de atendimento ao paciente, ou seja, um banco de dados capaz de reagir ao seu próprio conteúdo e não simplesmente um repositório em que, se o médico não atentar para as informações, nada de útil poderá obter. Vários mecanismos tem sido utilizados neste sentido já ao longo dos últimos 20 anos. A maioria destas técnicas é extremamente útil como mecanismo de suporte à decisão.

Apresentamos abaixo alguns exemplos sobre as possibilidades neste campo:

Lembretes automáticos - A característica básica que dá vida ao registro clínico computadorizado é a capacidade de executar automaticamente certos procedimentos com base nas informações que são armazenadas em seu banco de dados. Esta técnica, conhecida com o nome de triggering (gatilho) é algo até 
bastante conhecido ao nível de alguns dos bancos de dados relacionais comercialmente disponíveis. Sucintamente falando, especifica-se que, caso ocorra uma determinada condição, o computador deverá executar um determinado procedimento. Exemplo: todo o paciente com diagnóstico de hipertensão e que não tenha realizado uma consulta no último ano, deve ser convocado para comparecer ao hospital (a condição dispara um processo que gera a carta de convocação). No campo da medicina, foram inclusive criadas linguagens específicas para este fim como a MQL (Medical Query Language) do COSTAR (Computer Stored Ambulatory Register) e CARE do RMRS (Regenstrief Medical Record System).

Está embutido neste conceito o termo quality assurance, ou seja, o papel de lembrar ao médico quanto a uma série de providências ou até pistas diagnosticas ou de solicitação de exames com base em regras preestabelecidas ao nível da instituição. O sistema ideal deve ser suficientemente flexível para suportar regras introduzidas por diferentes níveis da organização, iniciando pelas regras embutidas em um pacote, provenientes do conhecimento médico geral, passando por regras do hospital, especialidade, enfermaria, até regras do próprio médico ou restritas ao paciente em questão. Note-se que isto pode envolver desde a lembrança de que se trata de uma doença de notificação compulsória (regra governamental) até lembretes específicos do que porventura tenha ficado combinado de detalhar com o paciente em sua próxima visita.

O emprego desta técnica também abre a possibilidade de não se ter de coletar todos os dados do paciente em uma única visita, dando assim maior flexibilidade ao médico. O computador, como instrumento capaz de ajudar a lembrar quanto a falta de dados importantes, garantiria, assim, o trabalho do médico.

Valoração das informações e capacidade de síntese - A possibilidade de grifar um resultado de exame laboratorial como anormal é um dos exemplos mais simples que demonstram a importância deste tipo de capacidade. Saber que informações são importantes e assim ressaltá-las para guiar o raciocínio clínico é uma atividade extremamente complexa. O valor de uma informação normalmente depende do contexto em que ela se enquadra (dos demais parâmetros) e também de sua localização na dimensão temporal (infarto há 10 anos é bem menos significativo do que na última semana). Contudo, esta capacidade resulta em extrema vantagem para o médico, quando empregada para gerar informações em um nível de abstração mais elevado, ou seja, a capacidade de sintetizar a informação. [4]

Presentemente, o aperfeiçoamento das tecnologias ópticas para armazenamento de dados (discos laser) poderá permitir realizar o sonho de ter todos os dados dos pacientes armazenados indefinidamente no sistema. Usando um disco CD-ROM (Compact Disk
Read Only Memory) com uma capacidade de 550 Mbytes, um manuscrito de 297.000 páginas de tamanho normal pode ser armazenado. A estrutura é flexível de tal forma que um "scanner" de imagem pode armazenar o "facsimile" de um registro ou filme de raio $\mathrm{X}$ ou um "scanner" de caracteres pode ser usado para converter cada caracter da página em código binário para armazenamento.

As implicações para isso são revolucionárias, uma vez que podem vir a resolver o problema de prontuários médicos não disponíveis ou perdidos, como também possibilitar a consulta a imagens de raio $\mathrm{X}$ e representações gráficas tal como ECG. A tecnologia do CD-ROM está disponível tanto para grandes Sistemas de Informações Hospitalares como também para micros departamentais. Histórias anteriores narram que a capacidade de armazenamento impressivo aumenta por fatores de aperfeiçoamento de tecnologia. A disponibilidade de tecnologia do CD-ROM torna possível o armazenamento de grandes bases locais assim como a conexão do Sistema de Informações Hospitalares a bancos de dados externos. [5]

Idealizou-se uma interligação entre os hospitais, laboratórios e postos de saúde, que permitiria o tráfego de informações dos Registros Clínicos. Via rede, cada local onde o paciente se apresentar, fornecendo um "código" mantido em cartão magnético, carteira plástica, placa metálica (como usam as forças armadas) permitiria o acesso aos dados clínicos.

\section{Exemplificando:}

Um paciente, vai a um posto de saúde do S.U.S. (Sistema Unificado de Saúde), e o médico atendente solicita exames de rotina do tipo, sangue, urina, fezes, radiologia e ambulatoriais. Recebidos os resultados (que já fazem parte do Registro Clínico Computadorizado), o paciente retorna a qualquer posto de saúde ou hospital interligado ao S.U.S., no que desta vez é atendido por outro plantonista, que solicita seu "código". O qual permitirá aceso aos exames já feitos anteriormente. Agilizando assim o atendimento ao paciente, reduzindo custos com duplicidade de exames e embasando tomadas de decisão.

Uma outra técnica de armazenamento que o Sistema de Informações Hospitalares pode ter é a preparação e elaboração de Smart-Card, um cartão de tamanho igual a um cartão de crédito codificado com dados demográficos do paciente assim como informações chaves sobre a saúde do paciente. Os dados no cartão devem conter informações a respeito de alergias, prescrição de drogas recentes, informações chaves de laboratório, assim como resultados dos procedimentos realizados. Se essa técnica pudesse ser utilizada daria a um profissional de atenção médica um panorama rápido dos cuidados médicos anteriores prestados ao paciente, eliminando a duplicação de exames e procedimentos. Existem, atualmente, cartões "inteligentes" baseados na 
em tecnologia óptica, que são capazes de armazenar até 200 Mbytes de informação em um único cartão. Dessa forma, é viável armazenar praticamente todo o prontuário do paciente, inclusive imagens médicas, traçados de sinais biológicos, resultados de exames de laboratório e demais textos associados ao processo da assistência. É o "prontuário médico de bolso", que poderá ser portado pelo paciente à medida que visita diferentes instituições de prestação de serviços de saúde. [5]

\section{Exemplos de Aplicações de Registro Clínico Computadorizado utilizando Smart-Card.}

Atualmente na França, $20 \%$ da população tem algum problema de rins. E sem o uso de máquinas de diálise certamente morreriam. Além disto, cada paciente gasta de 4 a 6 horas de três em três dias ligados a uma DIALYSIS (centro para diálise).

Cada DIALYSIS toma conta de dados complexos tal como dieta do paciente, estado de saúde, medicação e a diálise anterior. Recentemente o uso da informática tem simplificado a manipulação de tais informações. Há 245 centros de diálise na França. Até recentemente os pacientes só podiam ser tratados em sua " DIALYSIS ", porque suas informações vitais precisavam seguir seu tratamento médico, que não era armazenado de uma forma, onde poderia ser facilmente transferido.

Como resultado, muitos pacientes nunca puderam deixar sua casa para trabalhar ou divertir-se.

As placas inteligentes estão ajudando a mudar isto. Agora os pacientes são livres, transportando seus dados para qualquer centro de diálise, abrindo oportunidades novas para escolha do médico ou até viajar.

O cartão DIALYBRE, tem todas informações das últimas quinze diálises e prescrições anteriores, necessárias para decisões médicas no futuro. Estas informações somente poderão ser acessadas ou modificadas por um médico especializado, assim o paciente estará limitado a consultas com médicos treinados para trabalhar com este método. Cada paciente carrega consigo, em sua carteira, todas informações em formato que pode ser interpretado diretamente por computadores médicos, sem considerar onde o paciente esteve se tratando antes.

Cada DIALYBRE, tem informações médicas do tipo, grupo sangüíneo, fatores de compatibilidade de transplantes que podem ser acessados pelos médicos em caso de emergência. $\mathrm{O}$ cartão também possui dados para o acompanhamento das consultas como despesas de farmácia e ambulância, buscando assim reduzir custos e facilitar a vida do paciente reduzindo a burocracia e eliminando erros médicos. O sistema é novo e está sendo implantado na França, Espanha, Canadá e Alemanha.
Também na França, os "C3S" sistema usado pelo MUTUELLES DE FRANCE (Sociedade De seguro Mútuo da France), determinou por aplicar tecnologia de placa inteligente no campo de serviços sociais e saúde pública na France. A carta oferece usuários seus 3 tipos do serviço:

- um arquivo portátil de administração,

- um arquivo portátil médico,

- e condições de pagamento.

Na Alemanha, utiliza-se tecnologia Smart-Card na saúde pública.

As organizações de seguro na saúde pública, decidiram emitir placas inteligentes para mais de 70 milhões de pessoas, a fim de aperfeiçoar a eficiência administrativa e reduzir os custos do sistema. A escolha das placas inteligentes, faz parte de uma política nacional para erguer a qualidade dos serviços prestados na saúde pública e reduzir custos, aperfeiçoando a eficiência da manipulação da informação administrativa e médica. Os cartões utilizados, também contém informações como nome, endereço e detalhes da cobertura do seguro do paciente.

As placas inteligentes selecionam as informações, o acesso a cada item é limitado. Por exemplo:

Em caso de emergência a equipe de socorro tem acesso livre, sem precisar de permissão. Já o registro médico completo, só pode ser acessado por médicos qualificados para tal.

O emprego destes cartões reduz muito os erros e custos de manipulação de informações.

Estes cartões inteligentes, são do tamanho de cartões de crédito, plásticos, com um circuito integrado embutido. O circuito é responsável por manter as informações de forma eletrônica e controlar quem e como estas informações são usadas.

As informações contidas e o controle destas, dão origem a várias aplicações dos cartões inteligentes, o cartão têm impressas palavras e símbolos que podem ser compreendidos por quaisquer pessoas facilmente, e o circuito integrado guarda, seguramente informações que só podem acessadas por equipamentos eletrônicos. Esta combinação única, significa que a placa inteligente é uma ponte entre máquina e homem.

O cartão plástico tem seu tamanho determinado por padrões internacionais (ISO 7816). Este padrão também define o plástico e suas características físicas, tal como flexibilidade, tolerância a temperatura e posição dos contatos elétricos e suas funções e como o circuito integrado fala com o exterior.

As placas inteligentes, são assim chamadas por controlar o acesso às informações nelas contidas. Algumas placas somente permitem o acesso por senhas, isto significa que no cartão de saúde, o paciente, o médico, a administração do hospital, o seguro de saúde, cada um possui sua senha dando acesso a diferentes 
seções de informações. Em alguns casos não existem senhas, como por exemplo, as informações médicas de emergência tal como, grupo sangüíneo e nome do paciente.

\section{Tipos de acesso às informações:}

Somente para leitura. A informação é afixada como num livro, onde não pode ser adicionada, modificada ou apagada.

Somente para adição. O volume de informação só poderá ser aumentado. As informações não podem ser alteradas ou apagadas e só podem ser adicionadas até um determinado volume. Estas também poderão ser lidas.

Alteração e Deleção. Informações que poderão ser modificadas e/ou apagadas. Também dependerão de espaço em volume.

Sem acesso. Informações sem acesso, pode parecer inútil, mas as placas devem guardar segredos que nunca deverão ser vistos, como por exemplo as senhas.

\section{Conclusões}

Desde o desenvolvimento dos primeiros Sistemas de Informação Hospitalar tem havido o desejo de colocar neles a história clínica completa dos pacientes, para fins de recuperação individual ou em grupo, para pesquisa. $\mathrm{O}$ armazenamento de todos os dados demográficos, exames e resultados, mais as anotações da enfermagem e dos médicos, por vários anos, eleva a níveis proibitivos os custos do hardware do sistema, uma vez que tudo isso forma um volume imenso de informação, mesmo para hospitais pequenos. Os custos dos dispositivos de massa para armazenamento dos dados ainda são muito altos. Mesmo o armazenamento de apenas alguns dados clínicos relevantes é difícil, uma vez que não é fácil chegar a um consenso sobre que dados devem ser armazenados. O resultado disso é que o prontuário médico manuscrito continua sendo o documento primário para armazenamento sobre o paciente. [5]

Ao ver o estado de deploração, em que se encontra o Sistema de Saúde pública no Brasil, num primeiro momento acreditamos não haver mais como salvá-lo.

Mas pelo visto, não é a melhor conclusão. Acredito, ainda mais, que há solução e está no emprego de tecnologia. Está no fato de querer melhorar, sair da inércia, pois, ferramentas existem, e por incrível que pareça, no nosso caso viriam não só para facilitar a rotina administrativa, principalmente, mas para tentar impedir que dela se esgotassem os recursos financeiros que deveriam ser aplicados em sua própria melhora.

Repito, as ferramentas, a tecnologia do desenvolvimento e melhoria do sistema existem, e não são viabilizadas financeiramente, porque são desviadas pela aplicação incorreta do Governo Federal do Imposto sobre Operações Financeiras, senão pelo próprio Sistema Único de Saúde, em fraudes.

A implantação de um Sistema de Saúde Pública informatizado, melhor, moderno também esbarra naqueles que o impedem por interesses pessoais, pois a implantação de um sistema informatizado, aumentaria o controle administrativo e com ele a transparência das aplicações de recursos seria muito maior.

Esperamos que tenha ficado claro para o leitor que são inúmeras as necessidades de padronização se quisermos tirar proveito das informações armazenadas em computador, principalmente se o objetivo é implementar o registro clínico informatizado. Dentro do possível, deve-se evitar a criação de padrões locais e nacionais. É chegada a hora, portanto, de iniciarmos uma ampla discussão no sentido de adotar e adaptar os padrões existentes. Sugerimos que esta responsabilidade seja tomada por instituições em nível nacional, tais como a Sociedade Brasileira de Informática em Saúde. [3]

\section{Referências Bibliográficas}

[1].BLEICH, H. L. \& SLACK, W. V. Design of a Hospital Information System: A comparision between Interfaced and Integrated Systems. IN: Lun, K.C. et alii Eds. VII World Congress on Medical Informatics, Proceedings, Geneva, 6-10 Sept. 1992, North-Holland, Amsterdam, 1992, p. 174-177

[2].ILHA, J. O., O Registro Clínico Computadorizado no Hospital. Revista Informédica, 1(3):5-8, 1993.

[3].ILHA, J. O., O Registro Clínico Computadorizado: Padronização e Codificação. Revista Informédica, 1(5):5-8, 1993.

[4].ILHA, J. O., O Registro Clínico Computadorizado: Funções e Vantagens. Revista Informédica, 1(7):510, 1994.

[5].JOHANSTON, H., Sistemas de Informação Hospitalar: Presente e Futuro. Revista Informédica, 1(2):5-9, 1993

[6].WINFREE, R. G. et alii. Centrally Guided Distributed Information Systems: The Next Step. IN: Ball, M. J. et allii Eds. Healthcare Information Management Systems: A pratical guide. Springer Verlag, New York, p. 19-27, 1991 